\title{
Cost-effectiveness of sacubitril/valsartan for the treatment of patients with heart failure with reduced ejection fraction in Portugal
}

\author{
Margarida Borges ${ }^{a, b}$, Marta Afonso-Silva ${ }^{c}$, Pedro A. Laires ${ }^{c, d}$, Miguel Gouveiae, Joana Alarcãoa, Raquel Ascenção ${ }^{a, f}$ \\ and João Costa ${ }^{\mathrm{a}, \mathrm{b}}$

\begin{abstract}
aCenter for Evidence Based Medicine, Faculdade de Medicina, Universidade de Lisboa, Lisbon, Portugal; bLaboratório de Farmacologia Clínica e Terapêutica, Faculdade de Medicina, Universidade de Lisboa, Lisbon, Portugal; ' CHE\&OR, Novartis Farma, Produtos Farmacêuticos SA, Porto Salvo, Portugal; 'Centro de Investigação em Saúde Pública, Escola Nacional de Saúde Pública, Universidade NOVA de Lisboa, Lisbon, Portugal; ${ }^{\circ}$ Catolica Lisbon School of Business and Economics, Lisbon, Portugal; fClínica Universitária de Medicina Geral e Familiar, Faculdade de Medicina, Universidade de Lisboa, Lisbon, Portugal
\end{abstract}

\begin{abstract}
Objectives: This study assesses the cost-effectiveness of sacubitril/valsartan versus enalapril in patients with symptomatic heart failure with reduced ejection fraction (HFrEF).

Methods: We used a previously developed Markov model calibrated with patient-level data from the PARADIGM-HF trial, adapted to the Portuguese setting. The model considers two health states (alive or dead) and uses regression analyzes to estimate hospitalizations and deaths over time. A panel of experts estimated resource consumption in the outpatient setting. To estimate resource consumption with hospitalizations, the National Health Service Diagnosis Related Groups database was used. Unit costs were based on national legislation, and on the Infomed database. The model considers a societal perspective, a time horizon of 30-years, and a 5\% annual discount rate. Sensitivity analyses assessed the robustness of results.

Results: Sacubitril/valsartan increases life expectancy by 0.5 life-years, corresponding to 0.4 incremental quality adjusted life-years (QALY) versus enalapril. The estimated incremental cost-effectiveness ratio (ICER) is $22,702 € / Q A L Y$. Sensitivity analysis shows that results are robust, but sensitive to the parameter estimates of the cardiovascular survival curve.

Conclusion: Sacubitril/valsartan is a cost-effective therapeutic option in the treatment of Portuguese patients with HFrEF and translate into significant health gains and increased life expectancy versus the current standard of care.
\end{abstract}

ARTICLE HISTORY

Received 23 April 2019

Accepted 4 June 2019

\section{KEYWORDS}

Heart failure; costeffectiveness; angiotensinconverting enzyme inhibitors; angiotensin receptor neprilysin inhibitor; sacubitril/valsartan

\section{Introduction}

Heart failure (HF) is widely acknowledged as a global public health problem with high impact on patients' quality of life (QoL), hospitalizations, and mortality $[1,2]$.

The analysis of published international epidemiological data suggests an increase in the prevalence of $\mathrm{HF}$ in recent decades [3-5]. A Portuguese epidemiologic study published in 1998 (the EPICA study) estimated a HF prevalence of $4.4 \%$ (95\% confidence interval [Cl]: 3.7 to $5.0 \%$ ) in individuals older than 25 years [6]. A higher prevalence was observed in the older population, which may indicate that, due to the population ageing in recent decades, the current and future prevalence of $\mathrm{HF}$ in Portugal may be higher [7].

A recent burden of disease study estimated that HF accounts for $5 \%$ of the total deaths in mainland Portugal and for more than 20,000 disability-adjusted life years (DALY) [8]. Over the next two decades, as the population ages, the burden of $\mathrm{HF}$, measured in DALY, and the number of deaths due to HF are expected to increase by $28 \%$ and $73 \%$, respectively [8]. Furthermore, the $\mathrm{HF}$ burden has a significant economic impact in Portugal, estimated to be equivalent to $2.6 \%$ of the total public health expenditure [9].
The current recommended first-line treatments for patients with heart failure with reduced ejection fraction (HFrEF) with prior or current symptoms are angiotensin converting enzyme inhibitors (ACEI) or angiotensin receptor blockers (ARB) combined with a beta-blocker and a diuretic, as needed [10]. These agents have been shown to be cost-effective and in certain cases, besides reducing cardiovascular morbidity and mortality, are also cost-saving options [11-14].

Sacubitril/valsartan, a first-in-class angiotensin receptor neprilysin inhibitor (ARNI), is an innovative therapeutic option for the treatment of HFrEF. In the pivotal clinical trial, PARADIGM-HF, sacubitril/valsartan showed clinically relevant and statistically significant reduction in cardiovascular mortality and HF hospitalization after 27 months compared with enalapril (hazard ratio [HR] of $0.80,95 \% \mathrm{Cl}, 0.73$ to 0.87 ; p-value $<0.001$ ) [15]. In fact, sacubitril/valsartan is already recommended in the most recent guidelines for the treatment of patients with symptomatic HFrEF (New York Heart Association [NYHA] class II-IV) who tolerate an ACEI or ARB [10].

This study assesses the cost-effectiveness of sacubitril/ valsartan, for the treatment of patients with HFrEF with

CONTACT Marta Afonso-Silva marta.silva@novartis.com E HE\&OR, Novartis Farma, Produtos Farmacêuticos SA, Tagus Park, Avenida Professor Doutor Cavaco Silva n. ${ }^{\circ 10 E}$, Porto Salvo 2740-255, Portugal

(1) Supplemental data for this article can be accessed here

(c) 2019 Informa UK Limited, trading as Taylor \& Francis Group 
Article Highlights

- Heart failure is a major health problem worldwide, accounting for a significant social and economic burden.

- Sacubitril/valsartan, the first-in-class angiotensin receptor neprilysin inhibitor, is an innovative therapeutic option for the treatment of patients with symptomatic chronic heart failure with reduced ejection fraction.

- Sacubitril/valsartan showed clinically relevant and statistically significant mortality and heart failure hospitalization reductions versus enalapril in this population.

- This paper aims to assess the cost-effectiveness of sacubitril/valsartan compared with enalapril, both combined with standard therapy, for the treatment of patients with heart failure with reduced ejection fraction with New York Heart Association class II-IV in Portugal.

- Sacubitril/valsartan is estimated to be associated with health gains and increased life expectancy versus the current standard of care, which is translated into a cost-effective therapy for the treatment of patients with chronic heart failure with reduced ejection fraction.

NYHA class II-IV, compared to enalapril, in Portugal, adopting the societal perspective.

\section{Methods}

A previously developed Markov model [16] using patient-level data from the PARADIGM-HF trial [15] was adapted to the Portuguese setting.

The model considers two health states (alive or dead) and uses regression analyses to estimate hospitalizations and deaths over time. The model is based on predictive sub-models of cardiovascular mortality, hospitalization rate, and QoL, according to the treatment each patient receives and their clinical and demographic characteristics at baseline (Figure 1). This is a cohort based model which is run using the baseline characteristics and associated risks - of each patient in turn and the resulting outcomes recorded. Costs and outcomes are obtained for the cohort of patients as a whole by averaging individual costs and outcomes for all patients. The decision analytic model was constructed in $\mathrm{MS}^{\circledast}$ Excel $^{\circledast}$.

The simulation was run with one-month cycles, with halfcycle correction. Full details of the model have been reported previously [16].

Enalapril was chosen as the comparator not only because it was the active comparator in the PARADIGM-HF trial but also because the European Society of Cardiology (ESC) recommends the inclusion of an ACEI in the treatment of HFrEF and the Portuguese Society of Cardiology adopts the ESC guidelines [15,17].

Due to the absence of national data, the patient population was obtained from the PARADIGM-HF trial, i.e., adult patients with HFrEF and NYHA class II-IV, which in turn is the same population

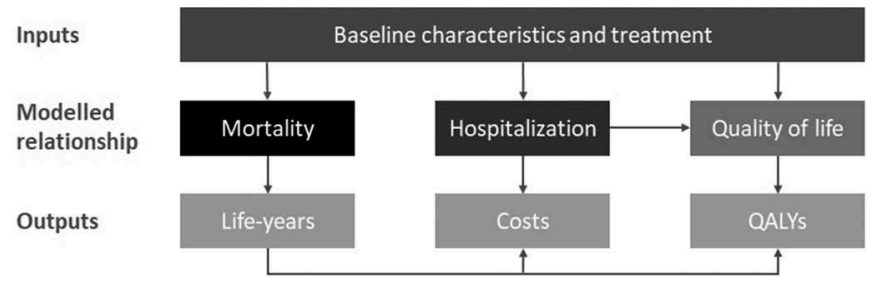

Figure 1. Conceptual model (based on McMurray et al., 2018 [16]).

Legend: QALYs, quality-adjusted life years. considered in sacubitril/valsartan label $[15,18]$. Detailed baseline characteristics are presented in Supplementary Table 1.

The societal perspective was adopted assuming a 30-year time horizon, during which all patients were expected to be dead. An annual 5\% discount rate was used for costs and consequences occurring after one year. This is aligned with the current Portuguese guidelines for health economic studies [19].

\subsection{Model inputs}

\subsubsection{Clinical data and health related quality of life}

Cardiovascular mortality, hospitalization rates, QoL, and adverse events (AE) were obtained from the PARADIGM-HF trial $[15,16]$. Details of the predictive sub-models used have been reported previously [16].

The cardiovascular mortality curve was estimated from the PARADIGM-HF data using the Gompertz parametric distribution to extrapolate beyond the duration of the trial. The Gompertz model resulted in a $\mathrm{HR}$ of $0.81(95 \% \mathrm{Cl}: 0.72$ to 0.90 ; $\mathrm{p}$-value<0.001) for sacubitril/valsartan versus enalapril, which is consistent with the primary statistical analysis of the PARADIGMHF (HR 0.80, 95\% Cl: 0.71 to 0.89; p-value<0.001). Details of the Gompertz model are presented in Supplementary Table 2.

Cardiovascular mortality depends on the treatment arm, patients' baseline characteristics, and time since randomization. The effect of hospitalization rates and $A E$ rates on cardiovascular mortality is implicitly included in the trial data $[15,16]$.

The non-cardiovascular mortality was estimated using the official Portuguese life tables by sex and age [20], adjusted to remove cardiovascular mortality. General and cardiovascular mortality in the Portuguese population was obtained from the Health Statistics 2013, published by Statistics Portugal [21]. Cardiovascular deaths were identified using the code LES - 33 (Diseases of the circulatory system). Cardiovascular mortality rates were converted into annual probabilities, assuming a constant risk. It was also assumed that the probability of death was constant in the 5-year age groups provided by Statistics Portugal. This probability of cardiovascular death was then subtracted from the general mortality in the life tables, resulting in the non-cardiovascular mortality.

The all-cause hospitalizations rate was also estimated from the PARADIGM-HF data using a negative binomial regression model permitting extrapolation beyond the end of the trial. The proportion of each hospitalization type is applied to estimate the number of HF, cardiovascular (non-HF) and non-cardiovascular hospitalizations experienced over the time horizon. These proportions are assumed to be constant over time. Details of the negative binomial regression model are presented in Supplementary Table 3.

Utility values were estimated using a mixed-effects model based on the treatment arm, baseline characteristics (including baseline EQ-5D), hospitalizations, AE, and time since randomization [16]. Supplementary Table 4 summarizes the results of the mixed-effects model. In addition to the differences in utilities between sacubitril/valsartan and enalapril due to differences in hospitalization rate and $A E$, sacubitril/valsartan was also associated with a small but statistically significant positive effect on EQ-5D, compared with enalapril, after adjusting for baseline characteristics, hospitalization, AE and time. Utilities used in the 
base-case scenario include this additional benefit of sacubitril/ valsartan. Also, the analysis of EQ-5D data suggested a utility decrease of 0.008 ( $95 \% \mathrm{Cl}: 0.006$ to 0.009 ) across both arms over the duration of the PARADIGM-HF trial (median follow-up of 27 months). In the absence of long-term EQ-5D data in patients with HFrEF, the base-case analysis assumed a constant decline in EQ-5D over the model's time horizon.

As it is expected that severe $A E$ are included in hospitalizations resources, the model considered only non-severe $A E$, particularly those pre-specified in the PARADIGM-HF trial which included hypotension, increase in serum creatinine, hyperkalemia, cough, and angioedema [15]. These AE were modeled assuming a constant incidence for each, as AE were assumed to have little impact on cost-effectiveness results (low cost, low incidence, and low impact on patients' QoL). AE incidence and mean duration were obtained from PARADIGM-HF (Supplementary Table 5) [15].

\subsubsection{Costs}

The following costs were considered: costs with therapy, HF management, inpatient care, medical visits and $A E$.

Pharmacological therapy costs were obtained from the National Authority of Medicines and Health Products (INFARMED) database (Infomed) from 2016 [22]. The cost of sacubitril/valsartan and enalapril was based on the average doses taken in the PARADIGM-HF trial (enalapril, $18.9 \mathrm{mg} /$ day and sacubitril/valsartan, 375 mg/day) [15]. Sacubitril/valsartan costs associated with titration consisted of two general medical practice visits. The standard therapy combined with either sacubitril/valsartan or enalapril included beta-blockers, diuretics, aldosterone antagonists, and digoxin. Additionally, due to the high usage observed in the PARADIGM-HF trial, anticoagulants, aspirin, adenosine diphosphate antagonists, and lipid lowering therapies were also considered. To estimate the standard therapy cost, the defined daily dose (DDD) was used and the dosage and package with the lowest price per mg were considered. The aggregated monthly cost of standard therapy, weighted by the percentage of patients who were taking it (based on the PARADIGM-HF trial [15]), was estimated at $11.7 €$. Other non-pharmacological therapy costs, such as implantable cardioverter-defibrillators, were considered to be included in the hospitalization and ambulatory costs.

To estimate resource consumption with hospitalizations, the National Health System 2014 Diagnosis related groups (DRG) Database was used [23]. According to the International Classification of Diseases, 9th Revision Clinical Modification (ICD9-CM), hospitalization episodes with the code 428.x (Heart Failure) were identified in any diagnosis. Of the episodes identified with $\mathrm{HF}$, those with a DDX1 (primary diagnosis) or a procedure code listed in Supplementary Table 6 were selected. The average unit cost of a HF patient hospitalization was estimated at $2,600 €$, by summing the products of the number of episodes in each selected DRG and their respective price, defined in the national legislation (Order 234/2015) [23,24].

The unit costs of health resources (medical visits, emergency episodes, and HF management) were obtained from national legislation (Order 234/2015) and their respective literature sources are detailed in Supplementary Table 7 [24].

A panel of Portuguese HF experts was undertaken to overcome the lack of information on resource consumption in the ambulatory setting. Experts were asked to give an estimation of the annual cost for the first and the following years with the disease (Table 1).

Given that the model does not permit a different cost for the first year and the following years, a value reflecting the average cost of the average life expectancy was estimated. Considering a mean survival of 6.5 years for a HFrEF patient (mean undiscounted life years of patients in sacubitril/valsartan and enalapril arms), an average monthly cost of $24 €$ was calculated for the healthcare resources consumed in the ambulatory setting (for both patients in sacubitril/valsartan and enalapril).

The healthcare resources consumption in the treatment of $A E$ associated with HF was defined by a panel of United Kingdom (UK) experts and validated by three of the Portuguese experts. Experts informed on the additional medical visits, medication, emergency room visits, blood tests or other health resources needed in the occurrence of an AE. Total cost per AE was calculated summing up the costs of the additional health resources identified (Table 2).

\subsection{Sensitivity analysis}

One-way deterministic sensitivity analyses were performed to test the robustness of the findings and evaluate the impact of uncertainty, considering alternative assumptions for key parameters in the model. Additional sensitivity analysis varying model's assumptions and modeling options were explored. The robustness of results was also assessed by probabilistic sensitivity analyses in which a total of 1,000 simulations were run.

\section{Results}

\subsection{Base case analysis}

Sacubitril/valsartan therapy potentially leads to an increased life expectancy of 0.52 life years (LY) when compared to enalapril. These health gains correspond to an incremental QALY of 0.44 versus enalapril (Table 3 ).

The total incremental cost difference between sacubitril/valsartan and enalapril was $10,021 €$ (Table 3 ). The most substantial cost difference was associated with the cost of primary drug

Table 1. Costs of healthcare resource consumption in the ambulatory setting obtained from the expert panel.

\begin{tabular}{lcc}
\hline & $\begin{array}{c}\text { Annual cost, } \\
1^{\text {st }} \text { year }\end{array}$ & $\begin{array}{c}\text { Annual cost, } \\
\text { following years }\end{array}$ \\
\hline Medical visits, specialty & $82 €$ & $41 €$ \\
Medical visits, GP & $78 €$ & $76 €$ \\
$\begin{array}{l}\text { Emergency room episodes (with no } \\
\quad \text { hospitalization) }\end{array}$ & $92 €$ & $42 €$ \\
Ambulance & $15 €$ & $8 €$ \\
Background medical management & $164 €$ & $97 €$ \\
Total & $\mathbf{4 3 2} €$ & $\mathbf{2 6 3} €$ \\
\hline
\end{tabular}

Legend: GP, general practice.

Table 2. Cost per adverse event.

\begin{tabular}{ll}
\hline Adverse event & Cost \\
\hline Hypotension & $62 €$ \\
Cough & $67 €$ \\
Angioedema & $89 €$ \\
Serum creatinine increase & $63 €$ \\
Hyperkalemia & $64 €$ \\
\hline
\end{tabular}


Table 3. Base case cost-effectiveness results estimated over lifetime (30 years), discounted.

\begin{tabular}{lccc}
\hline & Sacubitril/valsartan & Enalapril & $\Delta$ \\
\hline LY & 6.71 & 6.19 & 0.52 \\
QALY & 5.09 & 4.65 & 0.44 \\
Total costs & $19,949 €$ & $9,928 €$ & $10,021 €$ \\
ICER & & $22,702 €$ QQALY & \\
\hline
\end{tabular}

Legend: $\boldsymbol{\Delta}$, difference; ICER, incremental cost-effectiveness ratio; LY, life years; QALY, quality-adjusted life years.

therapy, which was partially offset by the reduction in hospitalization costs $(-543 €)$.

The incremental cost-effectiveness ratio (ICER) for sacubitril/ valsartan versus enalapril was 22,702 € per QALY gained (Table 3).

\subsection{Deterministic sensitivity analysis}

Scenario analyses were performed in which key structural assumptions were varied and ICERs reported. A total of 119 parameters were evaluated in the one-way sensitivity analysis. Results were robust in most scenarios (maximum ICER of 36,059 $€ / Q A L Y$ ), showing greater sensitivity to the parameters estimates of the cardiovascular survival curve (Figure 2). In fact, variations in the cardiovascular survival curve were the only scenarios tested with ICERs exceeding 30,000 €/QALY.

Additional sensitivity analyses were explored in which model assumptions or modeling options were altered. Table 4 shows the main results of this analysis. Results were robust but sensitive to the costs and consequences discount rate $(19,465$ $€ / Q A L Y$ when both costs and consequences discount rates are $0 \%)$ and when no additional benefit in patients' QoL with sacubitril/valsartan is assumed (27,065 €/QALY). Changes in other parameters or assumptions such as NYHA class, prior treatment with ACEI or ARB, $50 \%$ reduction in hospitalization costs, costs of sacubitril/valsartan and enalapril according to their targeted doses, among others, had no significant impact on the ICER (Table 4).

When an older population of patients was considered (mean $=67$ years), in line with the mean age of the sample in the EPICA study [6], and when the western European subgroup of patients in the PARADIGM-HF trial was also considered, an ICER of 20,534 €/QALY was obtained, showing the robustness of results to this parameter.

Results were sensitive to the time horizon. Nevertheless, sacubitril/valsartan remained cost-effective in a time horizon of 10 years with an ICER of $29,458 € /$ QALY.

\subsection{Probabilistic sensitivity analysis}

The cost-effectiveness scatterplot (Figure 3) shows that all simulations are in the northeast cost-effectiveness quadrant, showing that sacubitril/valsartan is more effective but costlier than enalapril. The estimated average probabilistic ICER was $24,281 € /$ QALY.

According to the probabilistic sensitivity analysis, $74 \%$ of the simulations resulted in an ICER $<30,000 € /$ QALY, the commonly accepted cost-effectiveness threshold in Portugal (Figure 4).

\section{Discussion}

$\mathrm{HF}$ is a global public health problem, with a high prevalence and social impact [1]. Despite the efficacy of therapeutic options available prior to the introduction of sacubitril/valsartan, there was undoubtedly a need to obtain greater benefits in terms of mortality and hospitalizations reduction, and signs and symptoms relief. Sacubitril/valsartan, a new treatment option for patients with HFrEF, showed clinically relevant and statistically significant reduction in mortality and hospitalizations [15].

The current cost-effectiveness study is the result of the adaptation of a previously developed model for the Portuguese reality, in which the cost-effectiveness of sacubitril/valsartan is compared with that of enalapril [16].

The base case results showed that besides its clinical benefit, sacubitril/valsartan also brings additional costs. However, compared to enalapril and given the commonly accepted Portuguese cost-effectiveness willingness-to-pay threshold of 30,000 $€ /$ QALY, sacubitril/valsartan is estimated to be cost-effective with an ICER of $22,702 \in /$ QALY. This result was mainly driven by a reduction in mortality and hospitalizations and by an improvement in QoL.

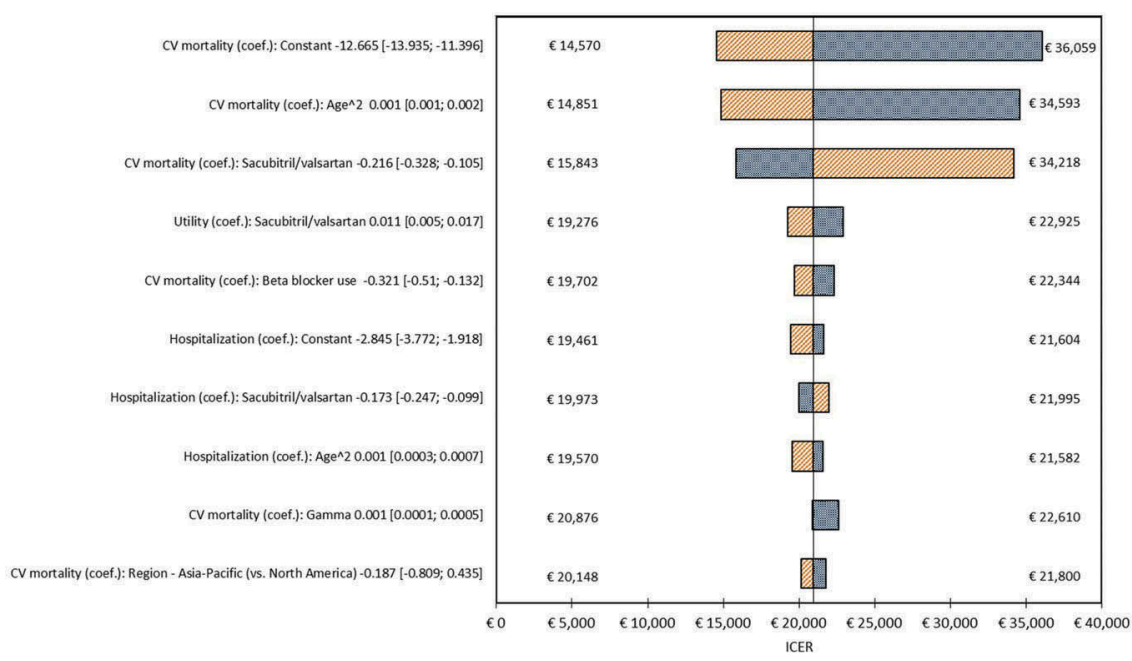

Figure 2. Tornado diagram with the 10 parameters that most influenced the ICER. Legend: coef., coefficient; CV, cardiovascular; ICER, incremental cost-effectiveness ratio. 
Table 4. Scenario analyses results.

\begin{tabular}{lc}
\hline Sensitivity analysis & ICER \\
\hline Base-case & $22,702 € / Q A L Y$ \\
No additional benefit of sacubitril/valsartan in utilities & $27,065 € / Q A L Y$ \\
Western Europe patients & $25,325 € / Q A L Y$ \\
All-cause mortality using data from PARADIGM-HF & $24,248 € / Q A L Y$ \\
Increase in non-CV mortality: SMR = 2 (instead of 1) ${ }^{\mathrm{a}}$ & $23,931 € / Q A L Y$ \\
Sacubitril/valsartan and enalapril costs according to & $23,351 € / Q A L Y$ \\
$\quad$ target doses & \\
50\% reduction in hospitalization costs & $23,317 € / Q A L Y$ \\
Baseline NYHA class III/IV & $22,967 € / Q A L Y$ \\
Prior use of ACEI & $22,873 € / Q A L Y$ \\
Baseline NYHA class I/II & $22,631 € / Q A L Y$ \\
Prior use of ARB & $22,131 € / Q A L Y$ \\
Discount rate (costs and consequences) $=0$ & $19,465 € / Q A L Y$ \\
\hline
\end{tabular}

Legend: $A C E l$, angiotensin converting enzyme inhibitors; $A R B$, angiotensin receptor blockers; CV, cardiovascular; ICER, incremental cost-effectiveness ratio; NYHA, New York Heart Association classification; QALY, quality-adjusted life years; SMR, standardized mortality ratio. ${ }^{a}$ standardized mortality ratios (SMR) are implemented in a scenario analysis such that non-cardiovascular mortality is increased by $100 \%$ in both model arms.

These results were robust to a range of sensitivity analyses, including the use of different sources for all-cause mortality, and a more advanced mean patient age. These analyses also showed that results were independent of disease severity or prior treatment with an ACEI or ARB which is representative of the Portuguese reality given that the reimbursed population are patients with HFrEF, NYHA II-III, and prior treatment for at least four weeks with an ACEI or ARB.

Comparison of these results with other countries is difficult due to country-specific information. Nevertheless, these findings are consistent with other recently published cost-effectiveness analyses in which sacubitril/valsartan is suggested to be a costeffective option for the treatment of HFrEF [16,25-30]. All studies reported an increase in life years with sacubitril/valsartan in all simulated populations. In the United States (US), sacubitril/valsartan demonstrated good value for money at the current acceptable threshold, with an ICER of 47,053 \$/QALY [30]. Likewise, in the UK, Colombia, and Denmark, sacubitril/valsartan was projected to yield cost-effectiveness ratios as low as 11,200 €/QALY (Colombia) and as high as 22,600 €/QALY (Denmark) when compared to enalapril, which are below the accepted costeffectiveness thresholds in each country [16]. In Germany, although cost-effectiveness analyses have no formal role in reimbursement and pricing of new pharmaceuticals, sacubitril/valsartan yielded an ICER of $26,278 € /$ QALY, which is at or below the ratio of other accepted interventions for the treatment of HF [25]. Additionally, an Italian study concluded that sacubitril/valsartan is a cost-effective option with an ICER of 19,487 €/QALY (below the usually accepted willingness-to-pay threshold of 40,000 $€ / Q A L Y$ in Italy) [28]. Differences in results between US and European studies are mainly due to differences in costs (i.e. the US has higher treatment and hospitalization costs [30]). For example, whereas in Portugal there is an increased cost of $10,021 €$ with sacubitril/valsartan versus enalapril, in the US, the incremental cost is $\$ 29,203$ [30].

This analysis is associated with some limitations. First, the clinical effectiveness of sacubitril/valsartan was based on a single clinical trial (PARADIGM-HF) that was stopped early due to a cardiovascular mortality benefit versus enalapril [15]. Also, the study population in the PARADIGM-HF was geographically diverse and may not fully represent the Portuguese patients. As costeffectiveness is dependent on patients' absolute risk of events, which is dependent on patients' baseline clinical characteristics, cost-effectiveness results may differ if PARADIGM-HF and Portuguese patients' characteristics do not align.

The adaptation of this model to the Portuguese reality consisted of using specific country input data whenever available. For non-cardiovascular mortality, Portuguese life tables were used. However, these data assumed that the non-cardiovascular health of a HF patient is similar to that of the general population. This may be inaccurate given that HF patients suffer from several comorbidities that may increase their risk of non-cardiovascular

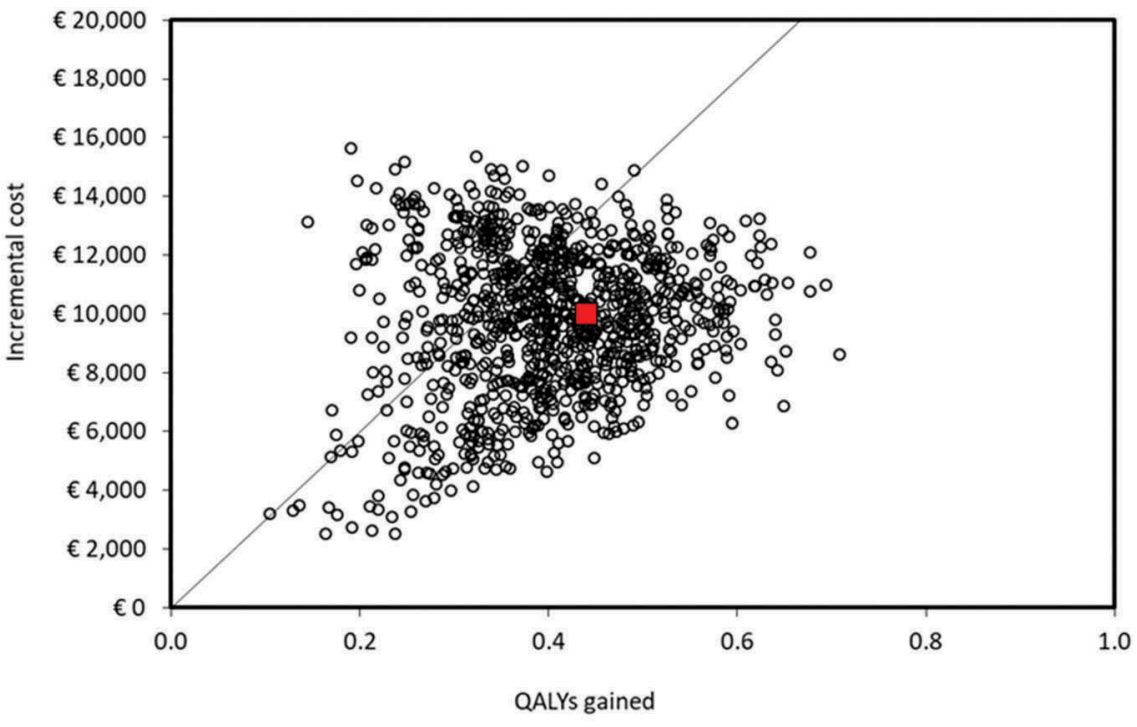

- Simulation result $\square$ Deterministic ICER — Linear (Threshold $(30,000 € /$ QALY))

Figure 3. Cost-effectiveness scatterplot (1,000 simulations).

Legend: QALYs, quality-adjusted life years; ICER, incremental cost-effectiveness ratio. 


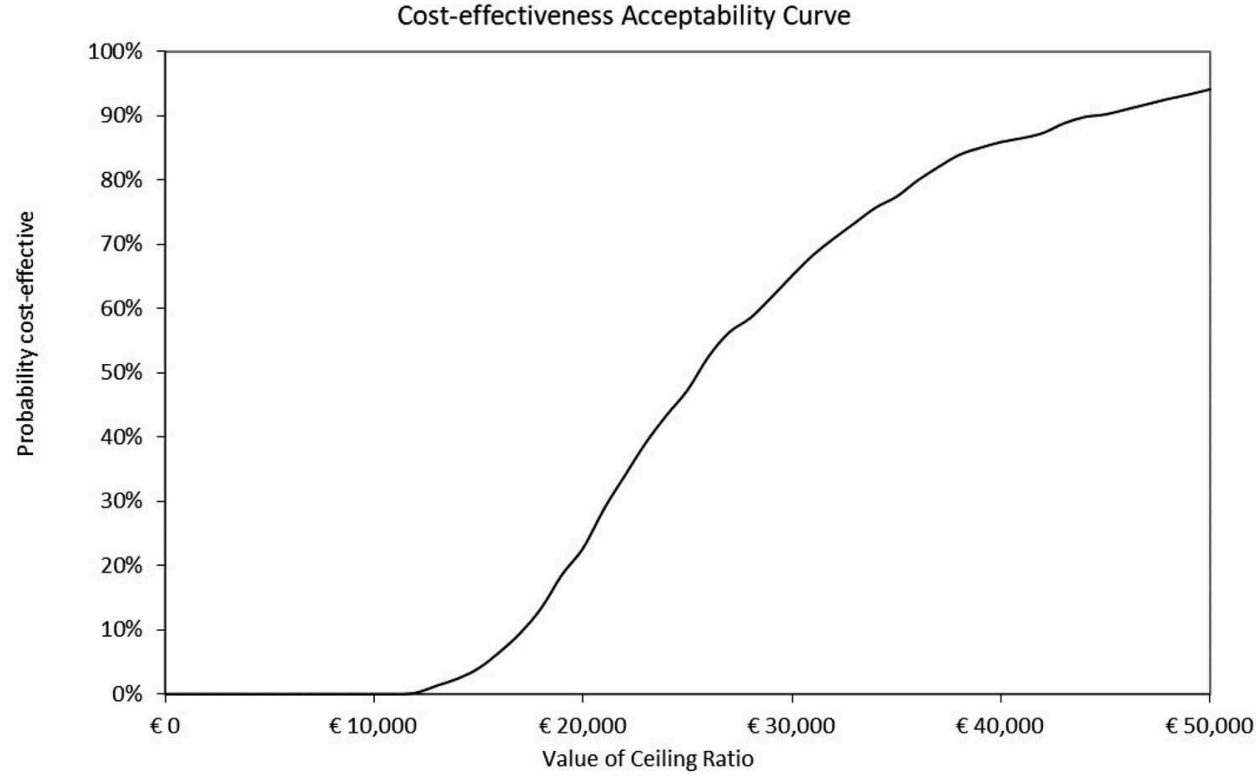

Figure 4. Cost-effectiveness acceptability curve.

mortality. To overcome this limitation, sensitivity analyses were run with the following changes: an increase in the noncardiovascular mortality by the application of different standardized mortality ratios ([SMR]; SMR = 2, ICER 23,931 €/QALY) and the utilization of the PARADIGM-HF data as the source of mortality for all causes (ICER 24,248 €/QALY). These results reinforced the robustness of the model.

Finally, despite being common practice in many costeffectiveness analyses, one of the major limitations of this study is the extrapolation of sacubitril/valsartan clinical outcomes beyond the PARADIGM-HF observation period. To address this, the long-term effects of treatment assumptions on mortality, QoL, and all-cause hospitalizations were examined by performing sensitivity analyses. Model results were most sensitive to the cardiovascular mortality and the hospitalizations curve; however, no ICER was above $36,059 € / Q A L Y$. As expected, results were sensitive to shorter time horizons as the greater benefit of sacubitril/valsartan is attributable to increased survival (for instance, for a time horizon of 10 years the ICER obtained was $29,458 € / Q A L Y$ ).

Several strengths of the current study should be noted. Firstly, patient-level data from the PARADIGM-HF [15] trial were used to predict cardiovascular mortality, all-cause hospitalization, and health-related QoL. This provided a high level of internal consistency as well as the opportunity to incorporate heterogeneity between patients in the model. Another strength was the way EQ-5D results were used and analyzed in the model. EQ-5D scores, from the PARADIGM-HF trial, were modelled using a mixed effects model, which takes into consideration time trends observed in the trial rather than a single point in time [16].

\section{Conclusions}

Sacubitril/valsartan offers good value for money in the treatment of Portuguese patients with chronic heart failure with reduced ejection fraction, at a willingness-to-pay threshold of $30,000 €$. Moreover, this innovative therapy has a favorable impact on patients' morbidity and mortality.

\section{Funding}

This analysis was funded by Novartis Farma, Produtos Farmacêuticos SA.

\section{Declaration of interest}

M Afonso-Silva and P A. Laires are employees of Novartis Farma, Produtos Farmacêuticos SA, Porto Salvo, Portugal. The authors have no other relevant affiliations or financial involvement with any organization or entity with a financial interest in or financial conflict with the subject matter or materials discussed in the manuscript apart from those disclosed.

\section{Reviewer Disclosures}

Peer reviewers on this manuscript have no relevant financial or other relationships to disclose.

\section{Author contribution statement}

M Gouveia and J Alarcão undertook the analysis of data and interpreted results. $M$ Borges, R Ascenção and J Costa contributed to the interpretation of the results. M Afonso-Silva and $\mathrm{P} A$. Laires contributed to the interpretation of the results and preparation of the manuscript. All authors read and approved the final manuscript.

\section{References}

Papers of special note have been highlighted as either of interest $(\cdot)$ or of considerable interest (•.) to readers.

1. Ponikowski $P$, Anker SD, AlHabib KF, et al. Heart failure: preventing disease and death worldwide. ESC Heart Fail. 2014;1(1):4-25.

2. Coelho R, Ramos S, Prata J, et al. Heart failure and health related quality of life. Clin Pract Epidemiol Ment Health. 2005;1:19.

3. Schocken DD, Arrieta MI, Leaverton PE, et al. Prevalence and mortality rate of congestive heart failure in the United States. J Am Coll Cardiol. 1992;20(2):301-306.

4. Hoes AW, Mosterd A, Grobbee DE. An epidemic of heart failure? Recent evidence from Europe. Eur Heart J. 1998;19(Suppl):L:L2-9.

5. Kannel WB, Ho K, Thom T. Changing epidemiological features of cardiac failure. Br Heart J. 1994;72(2 Suppl):S3-9. 
6. Ceia F, Fonseca C, Mota T, et al. Prevalence of chronic heart failure in Southwestern Europe: the EPICA study. Eur J Heart Fail. 2002;4 (4):531-539.

- This study shows the prevalence of heart failure in Portugal.

7. Instituto Nacional de Estatística IP. Censos 2011: resultados definitivos - Portugal. 2011.

8. Gouveia $M$, Ascenção $R$, Fiorentino $F$, et al. The current and future burden of heart failure in Portugal. ESC Heart Fail. 2019;6(2):254-261. [Epub ahead of print].

-. This study shows the current (2014) and future burden of heart failure in Portugal.

9. Fiorentino F, Ascenção R, Gouveia M, et al. The cost of illness of heart failure in Portugal. Value Health. 2017;20(9):A610.

- This study shows the current (2014) costs associated with heart failure in Portugal.

10. Yancy CW, Januzzi JL Jr., Allen LA, et al. 2017 ACC expert consensus decision pathway for optimization of heart failure treatment: answers to 10 pivotal issues about heart failure with reduced ejection fraction: a report of the American College of Cardiology task force on expert consensus decision pathways. J Am Coll Cardiol. 2018;71(2):201-230.

11. Szucs TD, Goedde M, Berger $K$, et al. [Cost effectiveness of ACE inhibition in therapy of chronic heart failure in Switzerland: evaluation based on the SOLVD study]. Schweiz Med Wochenschr. 1997; 127(29-30):1234-1241.

12. Antonanzas Villar F, Anton Botella F, Echevarria Echarri L. [Costeffectiveness analysis of the treatment with enalapril of chronic heart failure in Spain]. Gac Sanit. 1996;10(54):135-142.

13. Glick H, Cook J, Kinosian B, et al. Costs and effects of enalapril therapy in patients with symptomatic heart failure: an economic analysis of the Studies of Left Ventricular Dysfunction (SOLVD) Treatment Trial. J Card Fail. 1995;1(5):371-380.

14. Paul SD, Kuntz KM, Eagle KA, et al. Costs and effectiveness of angiotensin converting enzyme inhibition in patients with congestive heart failure. Arch Intern Med. 1994;154(10):1143-1149.

15. McMurray JJ, Packer M, Desai AS, et al. Angiotensin-neprilysin inhibition versus enalapril in heart failure. N Engl J Med. 2014;371 (11):993-1004.

- This is the pivotal clinical trial (PARADIGM-HF) of sacubitril/ valsartan.

16. Jjv M, Trueman D, Hancock E, et al. Cost-effectiveness of sacubitril/ valsartan in the treatment of heart failure with reduced ejection fraction. Heart. 2018;104(12):1006-1013.

-. This is the manuscript of the original cost-effectiveness model of sacubitril/valsartan from where the current study was adapted.
17. Yancy CW, Jessup M, Bozkurt B, et al. ACC/AHA/HFSA Focused Update of the 2013 ACCF/AHA guideline for the management of heart failure: a report of the american college of cardiology/American heart association task force on clinical practice guidelines and the heart failure society of America. J Am Coll Cardiol. 2017;70(6):776-803.

18. EMA. EU SPC Entresto, last update: 22/06/2018.2018.

19. INFARMED. Orientações metodológicas para estudos de avaliação económica de medicamentos; 1998.

20. Instituto Nacional de Estatística IP. Tábua Completa de Mortalidade para Portugal - 2011-2013; 2014.

21. Instituto Nacional de Estatística IP. Estatísticas da Saúde - 2013; 2015.

22. INFARMED. Infomed; 2016.

23. Administração Central do Sistema de Saúde ACSS IP.

24. Ministério da Saúde. Portaria 234/2015 de 7 de agosto. (Diário da República n. ${ }^{\circ}$ 153/2015, Série I de 2015-08-07, 2015).

25. Gandjour A, Ostwald DA. Sacubitril/Valsartan (LCZ696): a novel treatment for heart failure and its estimated cost effectiveness, budget impact, and disease burden reduction in Germany. PharmacoEconomics. 2018;36(10):1285-1296.

26. Ramos IC, Versteegh MM, de Boer RA, et al. Cost effectiveness of the angiotensin receptor neprilysin inhibitor sacubitril/valsartan for patients with chronic heart failure and reduced ejection fraction in the Netherlands: a country adaptation analysis under the former and current dutch pharmacoeconomic guidelines. Value Health. 2017;20(10):1260-1269.

27. van der Pol S, Degener F, Postma MJ, et al. An economic evaluation of sacubitril/valsartan for heart failure patients in the Netherlands. Value Health. 2017;20(3):388-396.

28. D'Angiolella LS, Cortesi PA, Pitotti C, et al. Sacubitril/valsartan in heart failure with reduced ejection fraction: cost and effectiveness in the Italian context. Eur J Heart Fail. 2017;19 (11):1551-1553.

29. Ademi Z, Pfeil AM, Hancock E, et al. Cost-effectiveness of sacubitril/ valsartan in chronic heart-failure patients with reduced ejection fraction. Swiss Med Wkly. 2017;147:w14533.

30. Sandhu AT, Ollendorf DA, Chapman RH, Pearson SD, Heidenreich PA. Cost-effectiveness of sacubitril-valsartan in patients with heart failure with reduced ejection fraction. Ann Intern Med. 2016;165(10):681-689.

31. Instituto Nacional de Emergência (INEM). Relatório de Atividades e Contas 2012; 2013.

32. Comissão para a Reavaliação da Rede Nacional de Emergência e Urgência C. Reavaliação da Rede Nacional de Emergência/Urgência. (Fev 2012. Reviewed in 2014). 